\title{
Chemoselectivity Switching in the Rhodium-Catalyzed Reactions of 4-Substituted-1-sulfonyl-1,2,3-triazoles with Allenols: Noticeable Differences between 4-Acyl- and 4-Aryl- Triazoles
}

\author{
Benito Alcaide, ${ }^{\mathrm{a} *}$ Pedro Almendros, ${ }^{\mathrm{b} *}$ Israel Fernández, ${ }^{\mathrm{c}}$ Teresa Martínez del Campo, ${ }^{\mathrm{a}}$ \\ Guillermo Palop, ${ }^{\mathrm{a}}$ Mireia Toledano-Pinedo, ${ }^{\mathrm{a}}$ and Patricia Delgado-Martínez ${ }^{\mathrm{d}, \S}$ \\ a Grupo de Lactamas y Heterociclos Bioactivos, Departamento de Química Orgánica I, Unidad Asociada al CSIC, \\ Facultad de Química, Universidad Complutense de Madrid, 28040 Madrid, Spain, E-mail: alcaideb@quim.ucm.es \\ b Instituto de Química Orgánica General, Consejo Superior de Investigaciones Científicas, IQOG-CSIC, Juan de la \\ Cierva 3, 28006 Madrid, Spain, E-mail: palmendros@iqog.csic.es \\ c Departamento de Química Orgánica I and Centro de Innovación en Química Avanzada (ORFEO-CINQA), Facultad de \\ Química, Universidad Complutense de Madrid, 28040 Madrid, Spain \\ d CAI Difracción de Rayos X, Facultad de Química, Universidad Complutense de Madrid, 28040 Madrid, Spain
}

Received: ((will be filled in by the editorial staff))

Supporting information for this article is available on the WWW under http://dx.doi.org/10.1002/adsc.200\#\#\#\#\#.((Please delete if not appropriate))

\begin{abstract}
Tunable chemoselectivity ( $O$ - versus $C$-attack) in the rhodium-catalyzed reactions of allenols with 4substituted-1-sulfonyl-1,2,3-triazoles has been achieved through the replacement of the 4 -aryl substituent by a 4 acetyl moiety.
\end{abstract}

Keywords: allenes; heterocycles; homogeneous catalysis; ketones; rhodium

1-Sulfonyl-1,2,3-triazole chemistry has merged as an excellent approach for the functionalization of unsaturated moieties and the construction of heterocyclic frameworks. ${ }^{[1]}$ This strategy has been built through the use of 4-aryl-1-sulfonyl-1,2,3triazoles as the source of $\alpha$-imino metal-carbenes, which may be viewed as donor/acceptor carbenoids. At the end of 2017, Miura and Murakami unveiled the chemistry of acceptor/acceptor carbenoids, which were generated from 4-acyl-1-mesyl-1,2,3-triazoles, for the functionalization of aromatic $\mathrm{C}\left(\mathrm{sp}^{2}\right)-\mathrm{H}$ bonds (Scheme 1a). ${ }^{[2]}$ Markedly contrasting results were obtained in comparison with donor/acceptor carbenoids. ${ }^{[3]}$ On the other hand, dramatic growth in the synthetic utility of allenes has been noticed in the last decade. ${ }^{[4]}$ Recently, Miura and Murakami have described the metal-catalyzed reaction of 4-aryl-1tosyl-1,2,3-triazoles with simple allenes to form pyrroles or $\alpha, \beta, \gamma, \delta$-unsaturated imines (Scheme 1b), ${ }^{[5]}$ while we reported the transannulation reaction of 4aryl-1-tosyl-1,2,3-triazoles with allenols to give 2pyrrolines (Scheme 1c) ${ }^{[6]}$ Based on these results, we believed that allenols might also act as an $O$ containing nucleophiles able to trap acceptor/acceptor carbenoids. Herein, we describe an unprecedented $O$ - attack/reorganization sequence between allenols and 4-acyl-1-tosyl-1,2,3-triazoles leading to 3-methylene5,6-dioxo-hept-1-enyl-4-amine derivatives (Scheme 1d).

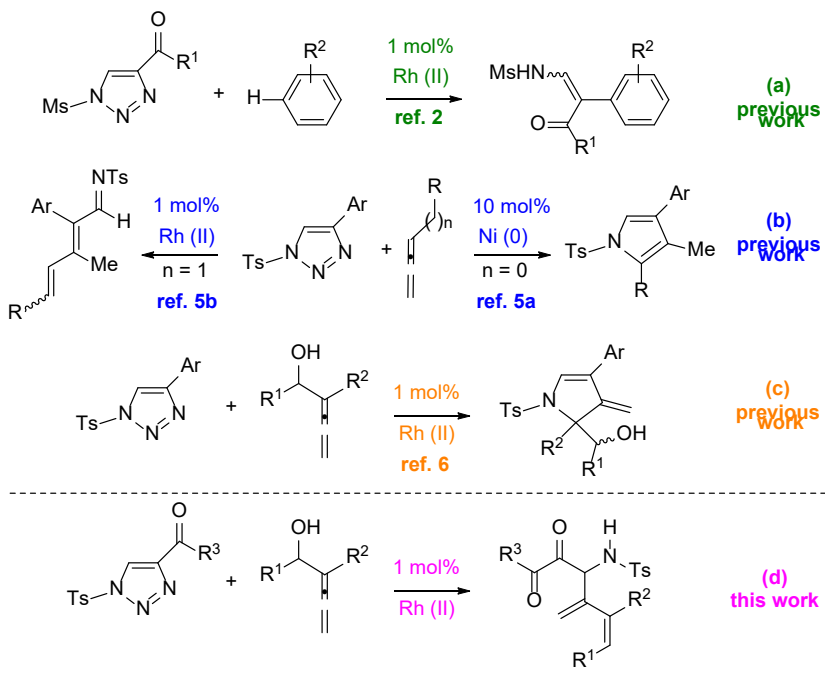

Scheme 1. Mode of reaction between 1-sulfonyl-1,2,3triazoles and allenes: previous work and the present study.

Allenol 1a was selected as model substrate. Initial experiments were performed through the reaction of 1a with the previously described 1-[1(methylsulfonyl)-1H-1,2,3-triazol-4-yl]ethan-1-one, but complex reaction mixtures were obtained. Nicely, 1-(1-tosyl-1H-1,2,3-triazol-4-yl)ethan-1-one 2, which was easily prepared following the reported procedure for its methylsulfonyl counterpart, ${ }^{[2]}$ was shown to be a promising reaction partner. The use of $\mathrm{Rh}_{2}(\mathrm{Oct})_{4}$ as 
catalyst ( $1 \mathrm{~mol} \%)$ and chloroform as solvent allowed the coupling between $1 \mathrm{a}$ and $\mathbf{2}$ to progress at $65^{\circ} \mathrm{C}$ and gave a reasonable $58 \%$ yield of product 3a (Table 1 , entry 3 ). The efficiency of the process could not be improved by moving to different metallic salts, solvents or reaction conditions. Adduct 3a is a polydecorated molecule ${ }^{[7]}$ bearing a 1,2-diketone functionality, a sulfonamide group, and a 1,3-diene moiety, which was obtained as single $Z$ isomer at the newly formed double bond. Noteworthy, replacing an aryl group by an acetyl group in the metal-catalyzed reactions of 4-substituted-1-sulfonyl-1,2,3-triazoles with allenols 1 dramatically provokes a chemoselectivity switch $\left(O\right.$ - versus $C$-attack). ${ }^{[8]}$

Table 1. Optimization of reaction conditions for the metalcatalyzed coupling of allenol $\mathbf{1 a}$ with acyl-triazole 2. ${ }^{[\mathrm{a}]}$, [b], [c]

\begin{tabular}{|c|c|c|c|c|c|}
\hline Entry & Catalyst & Solvent & $\begin{array}{c}\mathrm{T} \\
\left({ }^{\circ} \mathrm{C}\right)\end{array}$ & $\begin{array}{l}\text { Time } \\
\text { (h) }\end{array}$ & $\begin{array}{l}\text { Yield } \\
(\%)^{[\mathrm{d}]}\end{array}$ \\
\hline 1 & $\mathrm{Rh}_{2}(\mathrm{OAc})$ & $\mathrm{CHCl}_{3}$ & 65 & 2 & 3a (17) \\
\hline 2 & $\mathrm{Rh}_{2}(\mathrm{Esp})_{2}$ & $\mathrm{CHCl}_{3}$ & 65 & 2 & $3 \mathbf{a}(30)$ \\
\hline 3 & $\mathrm{Rh}_{2}(\mathrm{Oct})_{4}$ & $\mathrm{CHCl}_{3}$ & 65 & 2 & 3a (58) \\
\hline 4 & $\mathrm{Cu}(\mathrm{OTf})_{2}$ & toluene & 70 & 3 & - \\
\hline 5 & $\mathrm{AgNTf}_{2}$ & toluene & 70 & 4 & - \\
\hline 6 & $\mathrm{Rh}_{2}(\mathrm{Oct})_{4}$ & $\mathrm{CHCl}_{3}$ & 20 & 24 & $\mathbf{3 a}(14)$ \\
\hline 7 & $\mathrm{Rh}_{2}(\mathrm{Oct})_{4}$ & $\mathrm{CHCl}_{3}$ & 40 & 6 & 3a (35) \\
\hline 8 & $\mathrm{Rh}_{2}(\mathrm{Oct})_{4}$ & $\mathrm{CHCl}_{3}$ & 65 & 3 & 3a (49) \\
\hline 9 & $\mathrm{Rh}_{2}(\mathrm{Oct})_{4}$ & 1,2-DCE & 70 & 2 & $\mathbf{3 a}(45)$ \\
\hline 10 & $\mathrm{Rh}_{2}(\mathrm{Oct})_{4}$ & toluene & 70 & 8 & 3a (18) \\
\hline
\end{tabular}

[a] Reactions were carried out using 1a $(0.2 \mathrm{mmol}), 2$ (0.4 $\mathrm{mmol})$, and the catalyst $(0.002 \mathrm{mmol})$ in the corresponding solvent $(0.15 \mathrm{M})$.

[b] $\mathrm{OAc}=$ acetate; $\mathrm{Esp}=\left(\alpha, \alpha, \alpha^{\prime}, \alpha^{\prime}\right.$-tetramethyl-1,3benzenedipropionate); Oct $=$ octanoate; $1,2-\mathrm{DCE}=1,2-$ dichloroethane.

[c] 1,2-DCE = 1,2-dichloroethane.

[d] Yield of pure, isolated product with correct analytical and spectral data.

Having in hand the optimized conditions, we then investigated the scope and generality of the transformation by modifying the substitution on the allenol substrate 1 . The observed results are depicted in Scheme 2. Almost all reactions progressed smoothly to provide the desired products $\mathbf{3 a}-\mathbf{h}$ in reasonable yields. Various substituents on the aromatic ring $\left(\mathrm{R}^{1}\right)$ of different electronic demand such as activating (methyl and methoxy) and weakly deactivating (chlorine) were well accommodated. Different substitution patterns were also tolerated, because when a substituent was placed either at the ortho, meta, and para positions of the phenyl ring, the corresponding products were formed in fair yields as single $Z$ isomers. When the methyl group $\left(\mathrm{R}^{2}\right)$ of $\mathbf{1 a}$ was replaced by an aryl group such as phenyl and $4-\mathrm{BrC}_{6} \mathrm{H}_{4}$, the required polyfunctionalyzed adducts $\mathbf{3 f}-\mathbf{h}$ were efficiently obtained, without altering the total stereoselectivity. The single crystal XRD structure of adduct 3a unambiguously confirmed its polyfunctional nature and stereochemistry (Figure 1). ${ }^{[9]}$ Notably, the methylidene moiety survived under the reactions conditions and no isomerization towards the fully conjugated system occurred. Next, the scope of the reaction with respect to the 4-acyl-triazole reagent was explored using 1-(1-tosyl-1H-1,2,3-triazol-4yl)propan-1-one 2-Et and phenyl(1-tosyl-1H-1,2,3triazol-4-yl)methanone 2-Ph. Interestingly, triazole derivative 2-Et was as rewarding as triazole 2. As depicted in Scheme 2, the reactions of 2-Et with allenols $1 \mathbf{b}$ and 1c worked well for the obtention of molecules of type 3. Unfortunately, the Rh-catalyzed treatment of triazole 2-Ph with allenols $\mathbf{1 b}$ and $\mathbf{1 c}$ resulted in complicated reaction mixtures and was non-productive for the preparation of adducts 3 .

$$
\text { (1) }
$$

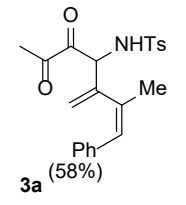
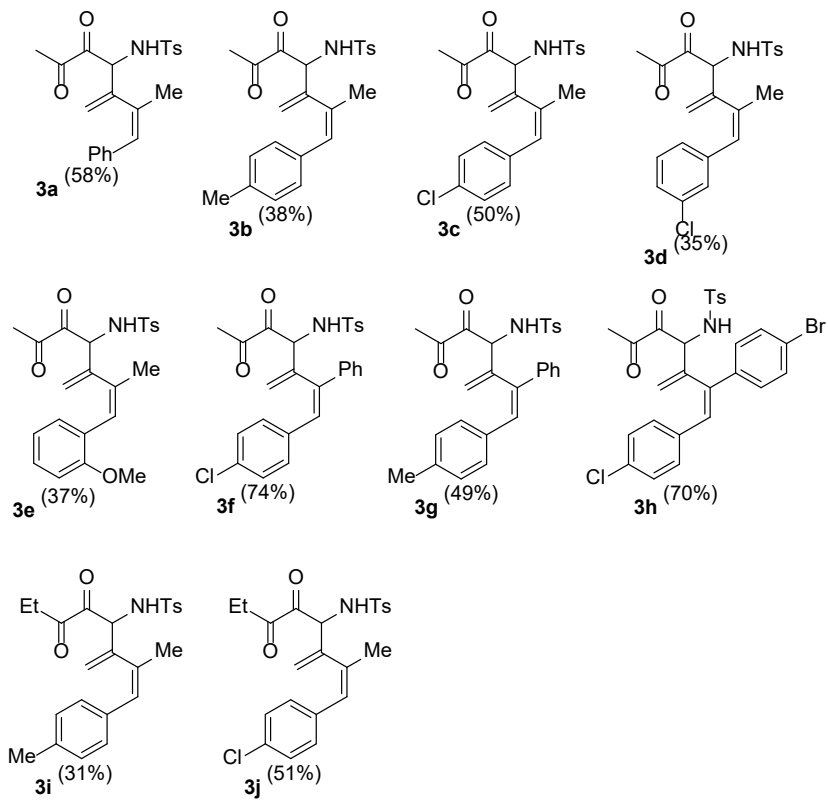

Scheme 2. Synthesis of polydecorated compounds $\mathbf{3 a}-\mathbf{j}$. 


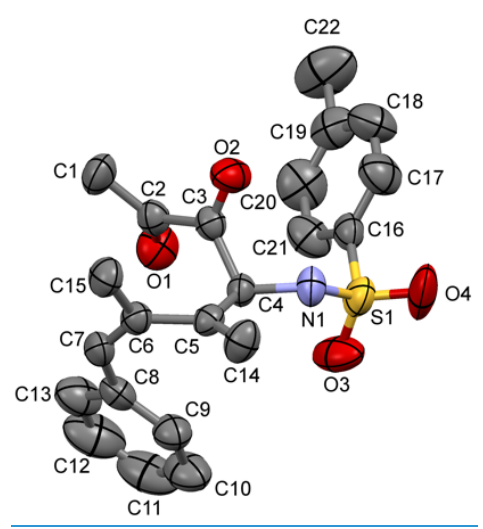

Figure 1. ORTEP drawing of (Z)-4-methyl- $N$-(2-methyl-3methylene-5,6-dioxo-1-phenylhept-1-en-4-

yl)benzenesulfonamide 3a. Thermal ellipsoids shown at $50 \%$ probability.

A possible pathway for the rhodium-catalysed generation of polyfunctional compounds 3 is outlined in Scheme 3. Initially, the formation of an $\alpha$-imino Rh(II)-carbenoid INT-2 may be involved. INT-2 should be formed by reaction of the $\mathrm{Rh}_{2}(\mathrm{Oct})_{4}$ catalyst with 4-acetyl-1-tosyl-1,2,3-triazole 2 through its $\alpha$ imino tautomer INT-1, with concurrent dinitrogen release, according to the general reaction mechanism proposed by Miura and Murakami. ${ }^{[2]}$ Next, nucleophilic addition of the hydroxyl functionality of allenes 1 to rhodacarbenoid INT-2 followed by deprotonation could lead to species INT-3. This path must be driven by the highly electrophilic character of the acceptor/acceptor rhodacarbenoid INT-2. ${ }^{[10]}$ Species INT-3 evolves into an allenyl vinyl ether intermediate after regeneration of the rhodium catalyst and concurrent protonation (Scheme 3). This allenyl vinyl ether intermediate may then undergo an allenic Claisen rearrangement to form 3-methylene-5,6dioxo-hept-1-enyl-4-amine derivatives 3 (Scheme 3), which resembles that proposed by Miura and Murakami. ${ }^{[7 b]}$

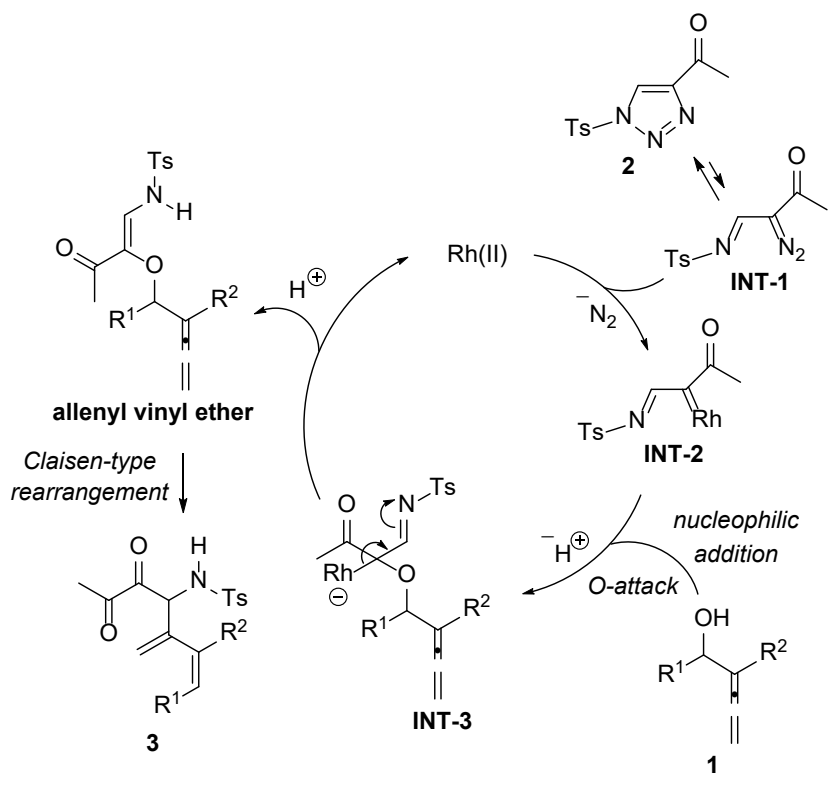

Scheme 3. Mechanistic explanation for the $\mathrm{Rh}(\mathrm{II})$-catalyzed synthesis of polyfunctional compounds $\mathbf{3}$.

Density Functional Theory (DFT) calculations at the dispersion-corrected $\mathrm{PCM}\left(\mathrm{CHCl}_{3}\right)$-B3LYPD3/def2-SVP level were carried out to gain more insight into mechanism involved in the transformation. ${ }^{[11]}$ To this end, the reaction between the experimentally used substrates allenol 1a and the $\mathrm{Rh}$ (II)-carbene INT-2 was explored.

As shown in Figure 2, which gathers the corresponding free energies (computed at $298 \mathrm{~K}$ ), the process begins with the formation of complex INT-4, where both reagents are linked by a hydrogen bond established between the $\mathrm{OH}$ group of the allenol and the nitrogen atom of the Rh(II)-carbenoid. INT-4 is then readily transformed into INT-5 by means of the insertion of the $\mathrm{OH}$ into the carbene carbon atom. This process is essentially barrierless, which is not surprising considering the highly exergonicity computed for this reaction step $\left(\Delta \mathrm{G}_{\mathrm{R}}=-45.9 \mathrm{kcal} / \mathrm{mol}\right)$. Indeed, relaxed scans from INT-4 at different $\mathrm{C}_{\text {carbene }} \cdots \mathrm{OH}$ confirms the barrierless nature of this insertion reaction (see Figure 3). Release of the weakly bonded dirhodium catalyst in INT-5 leads to zwitterion INT-6 in an endergonic transformation $\left(\Delta \mathrm{G}_{\mathrm{R}}=13.4 \mathrm{kcal} / \mathrm{mol}\right)$, which is compatible with the temperature required experimentally. ${ }^{[12]}$ As a result of the abstraction of a proton from the $\mathrm{OH}$-moeity by the imine, $(Z)$-allenyl vinyl ether INT-6 is stereoselectively formed. Finally, INT-6 can be directly converted into the observed dioxo-hept-1enyl-4-amine 3a through the transition state $\mathbf{T S}$ in a highly exergonic transformation $\left(\Delta \mathrm{G}_{\mathrm{R}}=-40.6\right.$ $\mathrm{kcal} / \mathrm{mol}$ ) and with a relatively low activation barrier of $15.2 \mathrm{kcal} / \mathrm{mol}$. As depicted in Figure 2, this saddlepoint is associated with the simultaneous $\mathrm{C}-\mathrm{O}$ bond rupture and formation of the new $\mathrm{C}-\mathrm{C}$ bond leading to the observed Z-dioxo-amine in a Claisen type [3,3]sigmatropic rearrangement. Therefore, our calculations are fully consistent with our initial 
proposal described above (Scheme 3) based on the proposal by Miura and Murakami. ${ }^{[\mathrm{b}]}$

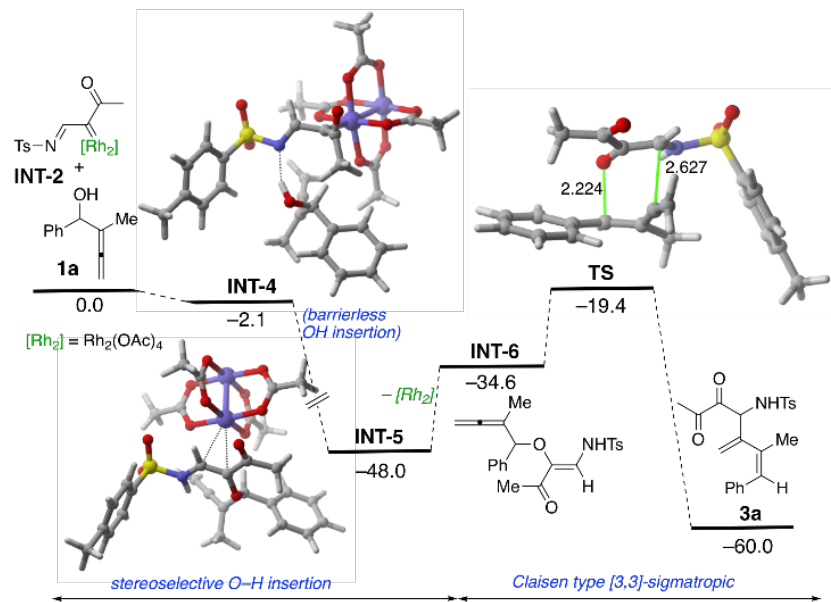

Figure 2. Computed profile for the transformation involving allenol 1a and $\mathrm{Rh}(\mathrm{II})$-carbene INT-2. Bond lengths and relative free energies $\left(\Delta \mathrm{G}_{298}\right.$, computed at 298 $\mathrm{K})$ are given in angstroms and $\mathrm{kcal} / \mathrm{mol}$, respectively. All data have been computed at the $\operatorname{PCM}\left(\mathrm{CHCl}_{3}\right)$-B3LYPD3/def2-SVP level.

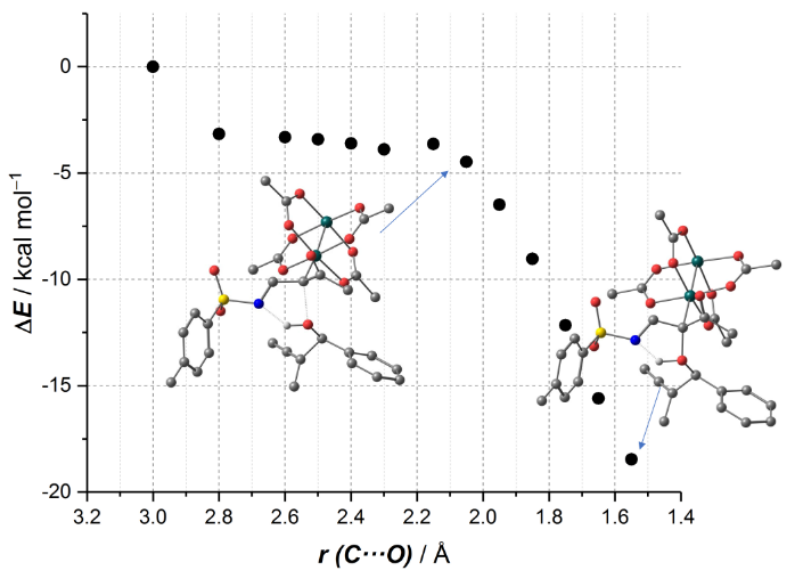

Figure 3. Relaxed scans modifying the key $\mathrm{C} \cdots \mathrm{O}$ distance for the INT-4 $\rightarrow$ INT-5 transformation. All data have been computed at the PCM(CHCl 3 -B3LYP-D3/def2-SVP level.

In conclusion, we have developed a divergent outcome transformation of the rhodium-catalyzed reactions of allenols with 4-substituted-1-sulfonyl1,2,3-triazoles, which has been accomplished through the replacement of the 4-aryl substituent by a 4-acetyl moiety. DFT calculations support the involvement of a $\mathrm{Rh}$ (II)-carbenoid produced from the reaction of the initial triazole and the dirhodium catalyst. This species is transformed, by reaction with the allenol, into an (Z)-allenyl vinyl ether intermediate which directly evolves into the observed reaction product through a Claisen type [3,3]-sigmatropic rearrangement.

\section{Experimental Section}

General methods: ${ }^{1} \mathrm{H}$ NMR and ${ }^{13} \mathrm{C}$ NMR spectra were recorded on a Bruker Avance-300 or Bruker Avance AMX700 spectrometers. NMR spectra were recorded in $\mathrm{CDCl}_{3}$ or $\mathrm{C}_{6} \mathrm{D}_{6}$, except otherwise stated. Chemical shifts are given in ppm relative to TMS $\left({ }^{1} \mathrm{H}, 0.0 \mathrm{ppm}\right), \mathrm{CDCl}_{3}\left({ }^{13} \mathrm{C}, 76.9 \mathrm{ppm}\right)$ and $\mathrm{C}_{6} \mathrm{D}_{6}\left({ }^{13} \mathrm{C}, 128.4 \mathrm{ppm}\right)$. Low and high resolution mass spectra were taken on an AGILENT 6520 Accurate-Mass QTOF LC/MS spectrometer using the electrospray mode (ES) unless otherwise stated. IR spectra were recorded on a Bruker Tensor 27 spectrometer. X-Ray crystallographic data were collected on a Bruker Smart CCD difractomer using graphite-monochromated Mo-K $\alpha$ radiation $(\lambda=$ $0.71073 \AA$ ) operating at $50 \mathrm{Kv}$ and $35 \mathrm{~mA}$ with an exposure of $30.18 \mathrm{~s}$ in $\omega$. All commercially available compounds were used without further purification.

General procedure for the rhodium-catalyzed reactions of allenols $1 \mathrm{a}-\mathrm{h}$ with acyl-triazole 2 . Synthesis of 3 methylene-5,6-dioxo-hept-1-enyl-4-amine derivatives 3a-h.

$\mathrm{Rh}_{2}(\mathrm{Oct})_{4}(2.3 \mathrm{mg}, 0.003 \mathrm{mmol})$ and acyl-triazole $2(159 \mathrm{mg}$, $0.6 \mathrm{mmol})$ were added to a stirred solution of the appropriate allenol $1(0.3 \mathrm{mmol})$ in chloroform $(2.0 \mathrm{~mL})$. The reaction mixture was stirred at $65{ }^{\circ} \mathrm{C}$ for $2 \mathrm{~h}$, allowed to cool to rt, and concentrated under reduced pressure. Chromatography of the residue eluting with hexanes/ethyl acetate (8:1) gave analytically pure compounds 3. Spectroscopic and analytical data for compounds $\mathbf{3}$ follow. ${ }^{[13]}$

(Z)-4-Methyl- $N$-(2-methyl-3-methylene-5,6-dioxo-1phenylhept-1-en-4-yl)benzenesulfonamide 3a. From 68 $\mathrm{mg}(0.427 \mathrm{mmol})$ of allenol 1a, and after chromatography of the residue, gave compound $\mathbf{3 a}(78 \mathrm{mg}, 58 \%)$ as a green solid; mp 103-105 ${ }^{\circ} \mathrm{C} ;{ }^{1} \mathrm{H}-\mathrm{NMR}\left(300 \mathrm{MHz}, \mathrm{C}_{6} \mathrm{D}_{6}, 25^{\circ} \mathrm{C}\right) \delta$ : $7.65(\mathrm{~d}, 2 \mathrm{H}, J=8.3 \mathrm{~Hz}, \mathrm{ArH}), 7.23$ (d, 2H, $J=7.3 \mathrm{~Hz}, \mathrm{ArH})$, 7.09 (t, 2H, $J=10.3 \mathrm{~Hz}, \mathrm{ArH}), 6.98(\mathrm{t}, 1 \mathrm{H}, J=7.3 \mathrm{~Hz}, \mathrm{ArH})$, $6.64(\mathrm{~d}, 2 \mathrm{H}, J=8.0 \mathrm{~Hz}, \mathrm{ArH}), 6.10(\mathrm{~s}, 1 \mathrm{H},=\mathrm{CH}), 5.72(\mathrm{~d}$, $1 \mathrm{H}, J=9.8 \mathrm{~Hz}, \mathrm{CH}-\mathrm{NH}), 5.59(\mathrm{~d}, 1 \mathrm{H}, J=9.8 \mathrm{~Hz}, \mathrm{CH}-\mathrm{NH})$, $5.03(\mathrm{~d}, 1 \mathrm{H}, J=1.2 \mathrm{~Hz}, \mathrm{CHH}), 4.91$ (s, 1H, CHH), 1.81 (d, $3 \mathrm{H}, J=1.4 \mathrm{~Hz}, \mathrm{Me}), 1.78$ (s, 3H, Me), 1.64 (s, 3H, Ar); ${ }^{13} \mathrm{C}-$ NMR (75 MHz, $\left.\mathrm{C}_{6} \mathrm{D}_{6}, 25^{\circ} \mathrm{C}\right) \delta: 195.5(\mathrm{C}=\mathrm{O}), 194.3(\mathrm{C}=\mathrm{O})$, 143.4, 143.2, 137.6, 137.1, 135.8, 129.7 (Ar, 2CH), 129.6 $(=\mathrm{CH}), 128.9(\mathrm{Ar}, 2 \mathrm{CH}), 128.5(\mathrm{Ar}, 2 \mathrm{CH}), 127,7(\mathrm{Ar}, 2 \mathrm{CH})$, $127.3(\mathrm{Ar}, \mathrm{CH}), 119.1\left(=\mathrm{CH}_{2}\right), 58.08(\mathrm{CH}-\mathrm{NH}), 26.08(\mathrm{Me})$, 23.4 (Me), 21.1 (Me); IR $\left(\mathrm{C}_{6} \mathrm{H}_{6}, \mathrm{~cm}^{-1}\right): v 3276,1718,1596$, 1159; HRMS (ES): calcd for $\mathrm{C}_{22} \mathrm{H}_{24} \mathrm{NO}_{4} \mathrm{~S}[M+\mathrm{H}]^{+}$: 398.14206; found: 398.14165 .

(Z)-4-Methyl-N-(2-methyl-3-methylene-5,6-dioxo-1-( $p$ tolyl)hept-1-en-4-yl)benzenesulfonamide $3 \mathrm{~b}$. From $50 \mathrm{mg}$ $(0.315 \mathrm{mmol})$ of allenol $\mathbf{1 b}$, and after chromatography of the residue, gave compound $\mathbf{3 b}(49 \mathrm{mg}, 38 \%)$ as a yellow oil; ${ }^{1} \mathrm{H}-\mathrm{NMR}\left(300 \mathrm{MHz}, \mathrm{C}_{6} \mathrm{D}_{6}, 25^{\circ} \mathrm{C}\right) \delta: 7.67(\mathrm{~d}, 2 \mathrm{H}, J=8.3 \mathrm{~Hz}$, ArH), 7.20 (s, 2H, ArH), 6.93 (d, 2H, J=7.9 Hz, ArH), 6.65 $(\mathrm{d}, 2 \mathrm{H}, J=8.0 \mathrm{~Hz}, \mathrm{ArH}), 6.10(\mathrm{~s}, 1 \mathrm{H},=\mathrm{CH}), 5.77(\mathrm{~d}, 1 \mathrm{H}, J$ $=9.7 \mathrm{~Hz}, \mathrm{CH}-\mathrm{NH}), 5.65$ (d, 1H, $J=9.8 \mathrm{~Hz}, \mathrm{CH}-\mathrm{NH}), 5.10$ $(\mathrm{d}, 1 \mathrm{H}, J=1.2 \mathrm{~Hz}, \mathrm{CHH}), 4.96(\mathrm{~s}, 1 \mathrm{H}, \mathrm{CH}), 2.06(\mathrm{~s}, 3 \mathrm{H}$, $\mathrm{Me}), 1.81$ (d, 3H, $J=1.4 \mathrm{~Hz}, \mathrm{Me}), 1.79$ (s, 3H, Me), $1.66(\mathrm{~s}$, $3 \mathrm{H}, \mathrm{Me}) ;{ }^{13} \mathrm{C}-\mathrm{NMR}\left(75 \mathrm{MHz}, \mathrm{C}_{6} \mathrm{D}_{6}, 25^{\circ} \mathrm{C}\right) \delta: 195.5(\mathrm{C}=\mathrm{O})$, $194.4(\mathrm{C}=\mathrm{O}), 143.4,143.3,137.7,136.9,134.9,134.3$, 
129.7 (Ar, 2CH), $129.6(\mathrm{Ar}, 2 \mathrm{CH}), 129.3(=\mathrm{CH}), 128.9(\mathrm{Ar}$, $2 \mathrm{CH}), 127,4$ (Ar. 2CH) $118.9\left(=\mathrm{CH}_{2}\right), 58.1(\mathrm{CH}-\mathrm{NH}), 26.2$ (Me), $23.4(\mathrm{Me}), 21.1(\mathrm{Me}), 21.1(\mathrm{Me})$; IR $\left(\mathrm{C}_{6} \mathrm{H}_{6}, \mathrm{~cm}^{-1}\right)$ : v 3272, 1718, 1600, 1160; HRMS (ES): calcd for $\mathrm{C}_{23} \mathrm{H}_{26} \mathrm{NO}_{4} \mathrm{~S}$ $[M+\mathrm{H}]^{+}:$412.15771; found: 412.15969 .

\section{(Z)-N-(1-(4-Chlorophenyl)-2-methyl-3-methylene-5,6-} dioxohept-1-en-4-yl)-4-methylbenzenesulfonamide $3 \mathrm{c}$. From $72 \mathrm{mg}(0.370 \mathrm{mmol})$ of allenol 1c, and after chromatography of the residue, gave compound $3 \mathbf{c}(78 \mathrm{mg}$, $50 \%)$ as a green solid; $\mathrm{mp} 106-108^{\circ} \mathrm{C} ;{ }^{1} \mathrm{H}-\mathrm{NMR}(300 \mathrm{MHz}$, $\left.\mathrm{C}_{6} \mathrm{D}_{6}, 25^{\circ} \mathrm{C}\right) \delta: 7.67(\mathrm{~d}, 2 \mathrm{H}, J=8.3 \mathrm{~Hz}, \mathrm{ArH}), 7.00(\mathrm{~m}, 4 \mathrm{H}$, ArH), 6.69 (d, 2H, $J=8.0 \mathrm{~Hz}, \mathrm{ArH}), 5.94$ (d, 1H, $J=1.0 \mathrm{~Hz}$, $=\mathrm{CH}), 5.88(\mathrm{~d}, 1 \mathrm{H}, J=9.8 \mathrm{~Hz}, \mathrm{CH}-\mathrm{N} H), 5.61(\mathrm{~d}, 1 \mathrm{H}, J=9.8$ $\mathrm{Hz}, \mathrm{CH}-\mathrm{NH}), 5.01(\mathrm{~d}, 1 \mathrm{H}, J=1.2 \mathrm{~Hz}, \mathrm{CHH}), 4.85$ (s, 1H, $\mathrm{CH} H$ ), 1.84 (s, 3H, Me), 1.79 (d, 3H, $J=1.5 \mathrm{~Hz}, \mathrm{Me}), 1.71$ (s, 3H, Me); ${ }^{13} \mathrm{C}-\mathrm{NMR}\left(75 \mathrm{MHz}, \mathrm{C}_{6} \mathrm{D}_{6}, 25{ }^{\circ} \mathrm{C}\right) \delta: 195.6$ $(\mathrm{C}=\mathrm{O}), 194.3(\mathrm{C}=\mathrm{O}), 143.6,142.9,137.3,136.5,135.5$, 133.0, 130.2 (Ar, 2CH), 129.8 (Ar, 2CH), 128.7 (Ar, 2CH), $128.3(=\mathrm{CH}), 127.7(\mathrm{Ar}, 2 \mathrm{CH}), 119.1\left(=\mathrm{CH}_{2}\right), 57.9(\mathrm{CH}-$ $\mathrm{NH}), 26.0(\mathrm{Me}), 23.5(\mathrm{Me}), 21.1(\mathrm{Me})$; IR $\left(\mathrm{C}_{6} \mathrm{H}_{6}, \mathrm{~cm}^{-1}\right): v$ 3276, 1717, 1595, 1159; HRMS (ES): calcd for $\mathrm{C}_{22} \mathrm{H}_{23} \mathrm{ClNO}_{4} \mathrm{~S}[M+\mathrm{H}]^{+}$: 432.10308; found: 432.10452 .

\section{(Z)- $\mathrm{N}$-(1-(3-Chlorophenyl)-2-methyl-3-methylene-5,6-} dioxohept-1-en-4-yl)-4-methylbenzenesulfonamide $3 d$. From $78 \mathrm{mg}(0.40 \mathrm{mmol})$ of allenol 1d, and after chromatography of the residue, gave compound $\mathbf{3 d}(58 \mathrm{mg}$, $35 \%$ ) as a green oil; ${ }^{1} \mathrm{H}-\mathrm{NMR}\left(300 \mathrm{MHz}, \mathrm{C}_{6} \mathrm{D}_{6}, 25{ }^{\circ} \mathrm{C}\right) \delta$ : $7.60(\mathrm{~d}, 2 \mathrm{H}, J=8.3 \mathrm{~Hz}, \operatorname{ArH}), 7.17$ (s, 1H, ArH), 7.02 (d, $1 \mathrm{H}, J=7.7 \mathrm{~Hz}, \mathrm{ArH}), 6.94(\mathrm{~d}, 1 \mathrm{H}, J=8.9 \mathrm{~Hz}, \mathrm{ArH}), 6.80(\mathrm{t}$, $1 \mathrm{H}, J=7.8 \mathrm{~Hz}, \mathrm{ArH}), 6.64$ (d, 2H, $J=8.0 \mathrm{~Hz}, \mathrm{ArH}), 5.89$ $(\mathrm{s}, 1 \mathrm{H},=\mathrm{CH}), 5.58(\mathrm{~d}, 1 \mathrm{H}, J=10.0 \mathrm{~Hz}, \mathrm{CH}-\mathrm{N} H), 5.36(\mathrm{~d}$, $1 \mathrm{H}, J=9.9 \mathrm{~Hz}, \mathrm{CH}-\mathrm{NH}), 4.85(\mathrm{~d}, 1 \mathrm{H}, J=1.2 \mathrm{~Hz}, \mathrm{CH} H)$, $4.78(\mathrm{~s}, 1 \mathrm{H}, \mathrm{CH} H), 1.80$ (s, 3H, Me), 1.77 (d, $J=1.5 \mathrm{~Hz}, 3 \mathrm{H}$, $\mathrm{Me}), 1.68$ (s, 3H, Me); ${ }^{13} \mathrm{C}-\mathrm{NMR}\left(75 \mathrm{MHz}, \mathrm{C}_{6} \mathrm{D}_{6}, 25^{\circ} \mathrm{C}\right) \delta$ : $195.5(\mathrm{C}=\mathrm{O}), 194.0(\mathrm{C}=\mathrm{O}), 143.5,142.8,139.0,137.6$, 137.5, 134.4, 129.8 (Ar, 2CH), $129.0(\mathrm{Ar}, \mathrm{CH}), 128.1(\mathrm{Ar}$, $\mathrm{CH}), 127.8(\mathrm{Ar}, \mathrm{CH}), 127.7(\mathrm{Ar}, \mathrm{CH}), 127.3(\mathrm{Ar}, 2 \mathrm{CH})$, 126.9 (Ar, CH), $119.4\left(=\mathrm{CH}_{2}\right), 58.0(\mathrm{CH}-\mathrm{NH}), 25.8(\mathrm{Me})$, $23.4(\mathrm{Me}), 21.1(\mathrm{Me})$; IR $\left(\mathrm{C}_{6} \mathrm{H}_{6}, \mathrm{~cm}^{-1}\right)$ : v 3273, 1718, 1596, 1160; HRMS (ES): calcd for $\mathrm{C}_{22} \mathrm{H}_{26} \mathrm{ClN}_{2} \mathrm{O}_{4} \mathrm{~S}\left[M+\mathrm{NH}_{4}\right]^{+}$: 449.12963; found: 449.13153 .

\section{(Z)-N-(1-(2-Methoxyphenyl)-2-methyl-3-methylene-} 5,6-dioxohept-1-en-4-yl)-4-methylbenzenesulfonamide 3e. From $72 \mathrm{mg}(0.378 \mathrm{mmol})$ of allenol 1e, and after chromatography of the residue, gave compound $3 \mathbf{e}(61 \mathrm{mg}$, $37 \%$ ) as a green oil; ${ }^{1} \mathrm{H}-\mathrm{NMR}\left(300 \mathrm{MHz}, \mathrm{C}_{6} \mathrm{D}_{6}, 25{ }^{\circ} \mathrm{C}\right) \delta$ : $7.65(\mathrm{~d}, 2 \mathrm{H}, J=8.3 \mathrm{~Hz}, \mathrm{ArH}), 7.32(\mathrm{dd}, 1 \mathrm{H}, J=7.5,1.1 \mathrm{~Hz}$, ArH), $7.01(\mathrm{t}, 1 \mathrm{H}, J=12.5 \mathrm{~Hz}, \mathrm{ArH}), 6.80(\mathrm{t}, 1 \mathrm{H}, J=7.4$ $\mathrm{Hz}, \mathrm{ArH}), 6.65$ (d, 2H, J=8.0 Hz, ArH), 6.50 (m, 2H, ArH $+=\mathrm{CH}), 5.64(\mathrm{q}, 2 \mathrm{H}, J=4.3 \mathrm{~Hz}, \mathrm{CH}-\mathrm{NH}), 4.97(\mathrm{~s}, 1 \mathrm{H}$, $\mathrm{CHH}), 4.93$ (s, 1H, CHH), 3.41 (s, 3H, OMe), 1.78 (s, 3H, $\mathrm{Me}), 1.77$ (d, 3H, $J=1.5 \mathrm{~Hz}, \mathrm{Me}), 1.73(\mathrm{~s}, 3 \mathrm{H}, \mathrm{Me}) ;{ }^{13} \mathrm{C}-$ NMR (75 MHz, $\left.\mathrm{C}_{6} \mathrm{D}_{6}, 25^{\circ} \mathrm{C}\right) \delta: 195.8(\mathrm{C}=\mathrm{O}), 194.4(\mathrm{C}=\mathrm{O})$, 157.2, 143.2, 143.1, 137.9, 136.1, 130.4 (Ar, CH), 129.7 (Ar, $2 \mathrm{CH}), 128.8(=\mathrm{CH}), 127.3(\mathrm{Ar}, 2 \mathrm{CH}), 126.5,125.8(\mathrm{Ar}, \mathrm{CH})$, $120.7(\mathrm{Ar}, \mathrm{CH}), 119.2\left(=\mathrm{CH}_{2}\right), 110.9(\mathrm{Ar}, \mathrm{CH}), 58.2(\mathrm{CH}-$ $\mathrm{NH}), 55.0(\mathrm{OMe}), 25.3(\mathrm{Me}), 23.5(\mathrm{Me}), 21.1$ (Me); IR $\left(\mathrm{C}_{6} \mathrm{H}_{6}, \mathrm{~cm}^{-1}\right): v 3270,1718,1597,1160 ;$ HRMS (ES): calcd for $\mathrm{C}_{23} \mathrm{H}_{26} \mathrm{NO}_{5} \mathrm{~S}[M+\mathrm{H}]^{+}$: 428.15262; found: 428.15404 .
(Z)-N-(1-(4-Chlorophenyl)-3-methylene-5,6-dioxo-2phenylhept-1-en-4-yl)-4-methylbenzenesulfonamide $3 f$. From $60 \mathrm{mg}(0.234 \mathrm{mmol})$ of allenol 1f, and after chromatography of the residue, gave compound $\mathbf{3 f}(85 \mathrm{mg}$, $74 \%)$ as a yellow oil; ${ }^{1} \mathrm{H}-\mathrm{NMR}\left(300 \mathrm{MHz}, \mathrm{C}_{6} \mathrm{D}_{6}, 25^{\circ} \mathrm{C}\right) \delta$ $7.58(\mathrm{~d}, 2 \mathrm{H}, J=8.3 \mathrm{~Hz}, \mathrm{ArH}), 7.23$ (d, 2H, $J=8.5 \mathrm{~Hz}, \mathrm{ArH})$, 7.09 (m, 4H, ArH), $7.01(\mathrm{~m}, 3 \mathrm{H}), 6.61(\mathrm{~d}, 2 \mathrm{H}, J=8.0 \mathrm{~Hz}$, $\mathrm{ArH}), 6.45(\mathrm{~s}, 1 \mathrm{H},=\mathrm{CH}), 5.78(\mathrm{~d}, 1 \mathrm{H}, J=9.8 \mathrm{~Hz}, \mathrm{CH}-\mathrm{NH})$, $5.69(\mathrm{~d}, 1 \mathrm{H}, J=9.8 \mathrm{~Hz}, \mathrm{CH}-\mathrm{NH}), 5.46(\mathrm{~d}, 1 \mathrm{H}, J=1.2 \mathrm{~Hz}$, $\mathrm{CHH}), 5.05(\mathrm{~s}, 1 \mathrm{H}, \mathrm{CH} H), 1.81(\mathrm{~s}, 3 \mathrm{H}, \mathrm{Me}), 1.51(\mathrm{~s}, 3 \mathrm{H}$, $\mathrm{Me}) ;{ }^{13} \mathrm{C}-\mathrm{NMR}\left(75 \mathrm{MHz}, \mathrm{C}_{6} \mathrm{D}_{6}, 25^{\circ} \mathrm{C}\right) \delta$ : $194.8(\mathrm{C}=\mathrm{O})$, $194.4(\mathrm{C}=\mathrm{O}), 143.5,140.4,140.1,139.9,137.3,135.1$, 133.8, 130.9 (Ar, 2CH), 130.5 (Ar, 2CH), 129.8 (Ar, 2CH), 128.9 (Ar, 2CH), 128,1 (Ar, CH), $128.0(=\mathrm{CH}), 127.7$ (Ar, 2CH), $127.4(\mathrm{Ar}, 2 \mathrm{CH}), 121.6\left(=\mathrm{CH}_{2}\right), 58.9(\mathrm{CH}-\mathrm{NH}), 23.1$ (Me), 21.1 (Me); IR $\left(\mathrm{C}_{6} \mathrm{H}_{6}, \mathrm{~cm}^{-1}\right):$ v 3263, 1719, 1596, 1159; HRMS (ES): calcd for $\mathrm{C}_{27} \mathrm{H}_{28} \mathrm{ClN}_{2} \mathrm{O}_{4} \mathrm{~S}\left[M+\mathrm{NH}_{4}\right]^{+}$: 511.14528; found: 511.14537 .

(Z)-4-Methyl- $N$-(3-methylene-5,6-dioxo-2-phenyl-1-( $p$ tolyl)hept-1-en-4-yl)benzenesulfonamide 3g. From $66 \mathrm{mg}$ $(0.279 \mathrm{mmol})$ of allenol $\mathbf{1 g}$, and after chromatography of the residue, gave compound $\mathbf{3 g}$ (64 $\mathrm{mg}, 49 \%$ ) as a yellow oil; ${ }^{1} \mathrm{H}-\mathrm{NMR}\left(300 \mathrm{MHz}, \mathrm{C}_{6} \mathrm{D}_{6}, 25^{\circ} \mathrm{C}\right) \delta: 7.58(\mathrm{~d}, 2 \mathrm{H} . J=8.2 \mathrm{~Hz}$, ArH), 7.44 (d, 2H, J=8.1 Hz, ArH), 7.19 (s, 1H, ArH), 7.02 $(\mathrm{dd}, 6 \mathrm{H}, J=9.5,7.9 \mathrm{~Hz}, \mathrm{ArH}), 6.66(\mathrm{~s}, 1 \mathrm{H},=\mathrm{CH}), 6.58(\mathrm{~d}$, $2 \mathrm{H}, J=8.1 \mathrm{~Hz}, \mathrm{ArH}), 5.82(\mathrm{~d}, 1 \mathrm{H}, J=9.8 \mathrm{~Hz}, \mathrm{CH}-\mathrm{N} H), 5.59$ (d, $1 \mathrm{H}, J=9.8 \mathrm{~Hz}, \mathrm{CH}-\mathrm{NH}), 5.52(\mathrm{~d}, 1 \mathrm{H}, J=1.1 \mathrm{~Hz}, \mathrm{CHH})$, 5.17 (s, 1H, CHH), 2.09 (s, 3H, Me), 1.77 (s, 3H, Me), 1.50 $(\mathrm{s}, 3 \mathrm{H}, \mathrm{Me}) ;{ }^{13} \mathrm{C}-\mathrm{NMR}\left(75 \mathrm{MHz}, \mathrm{C}_{6} \mathrm{D}_{6}, 25^{\circ} \mathrm{C}\right) \delta: 194.8$ $(\mathrm{C}=\mathrm{O}), 194.7(\mathrm{C}=\mathrm{O}), 143.2,140.7,140.5,138.4,137.9$, 137.5, 133.8, 131.9 (Ar, 2CH), 129.7 (Ar, 2CH), 129.6 (Ar, 2CH), $129.5(\mathrm{Ar}, 2 \mathrm{CH}), 128.8(=\mathrm{CH}), 128.3(\mathrm{Ar}, \mathrm{CH}), 127.7$ $(\mathrm{Ar}, 2 \mathrm{CH}), 127.6(\mathrm{Ar}, 2 \mathrm{CH}), 121.4\left(=\mathrm{CH}_{2}\right), 59.2(\mathrm{CH}-\mathrm{NH})$, $23.1(\mathrm{Me}), 21.2(\mathrm{Me}), 21.0(\mathrm{Me})$; IR $\left(\mathrm{C}_{6} \mathrm{H}_{6}, \mathrm{~cm}^{-1}\right):$ v 3279, 1718, 1598, 1159; HRMS (ES): calcd for $\mathrm{C}_{28} \mathrm{H}_{31} \mathrm{~N}_{2} \mathrm{O}_{4} \mathrm{~S}[M$ $\left.+\mathrm{NH}_{4}\right]^{+}:$491.19990; found: 491.19980 .

\section{(Z)-N-(2-(4-Bromophenyl)-1-(4-chlorophenyl)-3-} methylene-5,6-dioxohept-1-en-4-yl)-4-

methylbenzenesulfonamide $3 \mathrm{~h}$. From $64 \mathrm{mg}(0.19 \mathrm{mmol})$ of allenol $\mathbf{1 h}$, and after chromatography of the residue, gave compound $3 \mathbf{h}(69 \mathrm{mg}, 70 \%)$ as a yellow oil; ${ }^{1} \mathrm{H}-\mathrm{NMR}(300$ $\left.\mathrm{MHz}, \mathrm{C}_{6} \mathrm{D}_{6}, 25^{\circ} \mathrm{C}\right) \delta: 7.54(\mathrm{~d}, 2 \mathrm{H}, J=8.3 \mathrm{~Hz}, \mathrm{ArH}), 7.19$ (dd, $4 \mathrm{H}, J=5.8,2.8 \mathrm{~Hz}, \mathrm{ArH}), 7.09$ (m, 2H, ArH), 6.85 (m, $2 \mathrm{H}), 6.65$ (d, 2H, $J=8.0 \mathrm{~Hz}, \mathrm{ArH}), 6.34(\mathrm{~s}, 1 \mathrm{H},=\mathrm{CH}), 5.87$ $(\mathrm{d}, 1 \mathrm{H}, J=9.6 \mathrm{~Hz}, \mathrm{~N} H-\mathrm{CH}), 5.50(\mathrm{~d}, J=9.6 \mathrm{~Hz}, \mathrm{NH}-\mathrm{CH})$, $5.37(\mathrm{~d}, 1 \mathrm{H}, J=1.4 \mathrm{~Hz}, \mathrm{CHH}), 5.00(\mathrm{~d}, 1 \mathrm{H}, J=1.0 \mathrm{~Hz}$, $\mathrm{CH} H), 1.88$ (s, 3H, Me), 1.61 (s, 3H, Me); ${ }^{13} \mathrm{C}-\mathrm{NMR}(75$ $\left.\mathrm{MHz}, \mathrm{C}_{6} \mathrm{D}_{6}, 25^{\circ} \mathrm{C}\right) \delta: 195.1(\mathrm{C}=\mathrm{O}), 194.5(\mathrm{C}=\mathrm{O}), 143.7$, 139.9, 139.1, 138.9, 136.9, 134.7, 133.9, 131.9 (Ar, 2CH), $130.9(\mathrm{Ar}, 2 \mathrm{CH}), 130.8(=\mathrm{CH}), 129.8(\mathrm{Ar}, 2 \mathrm{CH}), 129.2(\mathrm{Ar}$, 2CH), 128.9 (Ar, 2CH), $127.65(\mathrm{Ar}, 2 \mathrm{CH}), 122.7,122.0$ $\left(=\mathrm{CH}_{2}\right), 58.7(\mathrm{CH}-\mathrm{NH}), 23.2(\mathrm{Me}), 21.2(\mathrm{Me}) ; \mathrm{IR}\left(\mathrm{C}_{6} \mathrm{H}_{6}\right.$, $\left.\mathrm{cm}^{-1}\right): \vee 3264,1718,2280,1596,1159$; HRMS (ES): calcd for $\mathrm{C}_{27} \mathrm{H}_{27} \mathrm{BrClN}_{2} \mathrm{O}_{4} \mathrm{~S}\left[M+\mathrm{NH}_{4}\right]^{+}$: 591.05379; found: 591.05528 .

(Z)-4-Methyl- $N$-(2-methyl-3-methylene-5,6-dioxo-1-( $p$ tolyl)oct-1-en-4-yl)benzenesulfonamide $3 i$. From $30 \mathrm{mg}$ $(0.172 \mathrm{mmol})$ of allenol $\mathbf{1 b}$, and after chromatography of the residue, gave compound $\mathbf{3 i}$ (22 $\mathrm{mg}, 31 \%$ ) as a yellow oil; 
${ }^{1} \mathrm{H}-\mathrm{NMR}\left(700 \mathrm{MHz}, \mathrm{C}_{6} \mathrm{D}_{6}, 25^{\circ} \mathrm{C}\right) \delta: 7.63(\mathrm{~d}, 2 \mathrm{H}, J=8.1 \mathrm{~Hz}$, $\operatorname{ArH}), 7.17$ (d, 2H, $J=8.0 \mathrm{~Hz}, \operatorname{ArH}), 6.92$ (d, 2H, $J=7.9$ $\mathrm{Hz}, \operatorname{ArH}), 6.61(\mathrm{~d}, 2 \mathrm{H}, J=7.9 \mathrm{~Hz}, \mathrm{ArH}), 6.09(\mathrm{~s}, 1 \mathrm{H},=\mathrm{CH})$, $5.76(\mathrm{~d}, 1 \mathrm{H}, J=9.8 \mathrm{~Hz}, \mathrm{CH}-\mathrm{N} H), 5.41(\mathrm{~d}, 1 \mathrm{H}, J=9.7 \mathrm{~Hz}$, $\mathrm{CH}-\mathrm{NH}), 5.07(\mathrm{~s}, 1 \mathrm{H},=\mathrm{CHH}), 4.94(\mathrm{~s}, 1 \mathrm{H},=\mathrm{CH} H), 2.23(\mathrm{~m}$, 1H, CHH), 2.05 (s, 3H, Me), 1.97 (m, 1H, CHH), 1.80 (m, $3 \mathrm{H}, \mathrm{Me}), 1.76(\mathrm{~s}, 3 \mathrm{H}, \mathrm{Me}), 0.67(\mathrm{t}, 3 \mathrm{H}, J=7.2 \mathrm{~Hz}, \mathrm{Me}) ;{ }^{13} \mathrm{C}-$ $\operatorname{NMR}\left(175 \mathrm{MHz}, \mathrm{C}_{6} \mathrm{D}_{6}, 25^{\circ} \mathrm{C}\right) \delta: 198.2(\mathrm{C}=\mathrm{O}), 194.5(\mathrm{C}=\mathrm{O})$, 143.4, 143.2, 137.7, 136.9, 134.9, 134.3, 129.7 (Ar, 2CH), $129.6(\mathrm{Ar}, 2 \mathrm{CH}), 129.3(=\mathrm{CH}), 129.0(\mathrm{Ar}, 2 \mathrm{CH}), 127,7$ (Ar, $2 \mathrm{CH}), 118.9\left(=\mathrm{CH}_{2}\right), 58.4(\mathrm{CH}-\mathrm{NH}), 29.9\left(\mathrm{CH}_{2}\right), 26.2(\mathrm{Me})$, $21.1(\mathrm{Me}), 21.0(\mathrm{Me}), 6.7(\mathrm{Me})$; IR $\left(\mathrm{C}_{6} \mathrm{H}_{6}, \mathrm{~cm}^{-1}\right): v 3662$, 2923, 1721, 1164; HRMS (ES): calcd for $\mathrm{C}_{24} \mathrm{H}_{28} \mathrm{NO}_{4} \mathrm{~S}[M+$ $\mathrm{H}]^{+}$: 426.17336; found: 426.17256 .

\section{(Z)- $N$-(1-(4-Chlorophenyl)-2-methyl-3-methylene-5,6-} dioxooct-1-en-4-yl)-4-methylbenzenesulfonamide $3 \mathbf{j}$. From $30 \mathrm{mg}(0.154 \mathrm{mmol})$ of allenol $1 \mathrm{c}$ and after chromatography of the residue, gave compound $\mathbf{3 j}$ ( $35 \mathrm{mg}$, $51 \%$ ) as a yellow oil; ${ }^{1} \mathrm{H}-\mathrm{NMR}\left(700 \mathrm{MHz}, \mathrm{C}_{6} \mathrm{D}_{6}, 25^{\circ} \mathrm{C}\right) \delta$ : $7.62(\mathrm{~d}, 2 \mathrm{H}, J=8.2 \mathrm{~Hz}, \mathrm{ArH}), 7.03$ (d, 2H, $J=8.5 \mathrm{~Hz}, \mathrm{ArH})$, $6.98(\mathrm{~d}, 2 \mathrm{H}, J=8.5 \mathrm{~Hz}, \mathrm{ArH}), 6.64$ (d, 2H, $J=8.0 \mathrm{~Hz}, \mathrm{ArH})$, $5.91(\mathrm{~s}, 1 \mathrm{H},=\mathrm{CH}), 5.60(\mathrm{~d}, 1 \mathrm{H}, J=9.9 \mathrm{~Hz}, \mathrm{CH}-\mathrm{NH}), 5.56$ (d, $1 \mathrm{H}, J=9.9 \mathrm{~Hz}, \mathrm{CH}-\mathrm{NH}), 4.96(\mathrm{~s}, 1 \mathrm{H},=\mathrm{CHH}), 4.82$ (s, $1 \mathrm{H},=\mathrm{CH} H), 2.26(\mathrm{~m}, 1 \mathrm{H}, \mathrm{CH} H), 2.03(\mathrm{~m}, 1 \mathrm{H}, \mathrm{CHH}), 1.80$ (s, 3H, Me), 1.77 (d, 3H, $J=1.4 \mathrm{~Hz}, \mathrm{Me}), 0.68$ (t, 3H, $J=$ $7.2 \mathrm{~Hz}, \mathrm{Me}) ;{ }^{13} \mathrm{C}-\mathrm{NMR}\left(175 \mathrm{MHz}, \mathrm{C}_{6} \mathrm{D}_{6}, 25^{\circ} \mathrm{C}\right) \delta: 198.3$ $(\mathrm{C}=\mathrm{O}), 194.4(\mathrm{C}=\mathrm{O}), 143.5,143.0,137.4,136.6,135.5$, 133.0, 130.2 (Ar, 2CH), $129.8(\mathrm{Ar}, 2 \mathrm{CH}), 128.7(=\mathrm{CH})$, 128.4 (Ar, 2CH), 127,7 (Ar, 2CH), $119.1\left(=\mathrm{CH}_{2}\right), 58.1(\mathrm{CH}-$ $\mathrm{NH}), 30.0\left(\mathrm{CH}_{2}\right), 26.1(\mathrm{Me}), 21.1(\mathrm{Me}), 6.7(\mathrm{Me}) ; \mathrm{IR}\left(\mathrm{C}_{6} \mathrm{H}_{6}\right.$, $\left.\mathrm{cm}^{-1}\right): v$ 3272, 1721, 1598, 1163; HRMS (ES): calcd for $\mathrm{C}_{23} \mathrm{H}_{28} \mathrm{ClN}_{2} \mathrm{O}_{4} \mathrm{~S}\left[M+\mathrm{NH}_{4}\right]^{+}$: 463.14528; found: 463.14539 .

\section{Acknowledgements}

Support for this work by the MINECO and FEDER (Projects CTQ2015-65060-C2-1-P, CTQ2015-65060-C2-2-P, and CTQ2016-78205-P and CTQ2016-81797-REDC) is gratefully acknowledged. G. P. and M. T.-P. thank CAM and FEDER (YEI) for predoctoral contracts.

\section{References}

$\S$ Responsible for X-ray crystal-structure determination.

[1] For representative reviews, see: a) Y. Li, H. Yang, H. Zhai, Chem. Eur. J. 2018, 24, 12757; b) Y. Jiang, R. Sun, X.-Y. Tang, M. Shi, Chem. Eur. J. 2016, 22, 17910; c) Y. Wang, X. Lei, Y. Tang, Synlett 2015, 26, 2051; d) H. M. L. Davies, J. S. Alford, Chem. Soc. Rev. 2014, 43, 5151 ; e) P. Anbarasan, D. Yadagiri, S. Rajasekar, Synthesis 2014, 46, 3004; f) A. V. Gulevich, V. Gevorgyan, Angew. Chem. Int. Ed. 2013, 52, 1371.

[2] T. Miura, Q. Zhao, M. Murakami, Angew. Chem. Int. Ed. 2017, 56, 16645.

[3] Li et al. recently reported the $\mathrm{Rh}(\mathrm{III})$-catalyzed $\mathrm{C}-\mathrm{C}$ coupling of arenes and 4-acyl-1-sulfonyltriazoles: $\mathrm{M}$. Tian, B. Liu, Ji. Sun, X. Li, Org. Lett. 2018, 20, 4946. [4]a) B. Yang, Y. Qiu, J.-E. Backvall, Acc. Chem. Res. 2018, 51, 1520; b) H.-U. Reissig, R. Zimmer, Synthesis 2017, 49, 3291; c) A. Lledó, A. Pla-Quintana, A. Roglans, Chem. Soc. Rev. 2016, 45, 2010; d) J. M. Alonso, M. T. Quirós, M. P. Muñoz, Org. Chem. Front. 2016, 3, 1186; e) W. Yang, A. S. K. Hashmi, Chem. Soc. Rev. 2014, 43, 2941; f) S. Kitagaki, F. Inagaki, C. Mukai, Chem. Soc. Rev. 2014, 43, 2956; g) S. Yu, S. Ma, Angew. Chem. Int. Ed. 2012, 51, 3074 .

[5] a) T. Miura, K. Hiraga, T. Biyajima, T. Nakamuro, M. Murakami, Org. Lett. 2013, 15, 3298; b) T. Miura, T. Nakamuro, T. Biyajima, M. Murakami, Chem. Lett. 2015, 44, 700 .

[6] B. Alcaide, P. Almendros, S. Cembellín, T. Martínez del Campo, G. Palop, Chem. Eur. J. 2017, 23, 13754.

[7] For the synthesis of 2-substituted 2-amino ketones by rhodium-catalyzed reaction of $N$-sulfonyl-1,2,3triazoles with 2-alkenols, see: a) T. Miura, T. Tanaka, Q. Zhao, S. G. Stewart, M. Murakami, Helv. Chem. Acta 2017, 100, e1600320. For the one-pot introduction of three different bonds onto terminal alkynes through $\mathrm{N}$ sulfonyl-1,2,3-triazole intermediates, see: b) T. Miura, T. Tanaka, T. Biyajima, A. Yada, M. Murakami, Angew. Chem. Int. Ed. 2013, 52, 3883.

[8] The preparation of different cores through divergent reactivity is normally catalyst- or ligand-directed. For selected reviews, see: a) V. Michelet, P. Y. Toullec, J.P. Genet, Angew. Chem. Int. Ed. 2008, 47, 4268; b) J. Mahatthananchai, A. M. Dumas, J. W. Bode, Angew. Chem. Int. Ed. 2012, 51, 10954; c) Y.-C. Lee, K. Kumar, H. Waldmann, Angew. Chem. Int. Ed. 2018, 57, 5212.

[9] CCDC-1839876 contains the supplementary crystallographic data for this paper.

[10] In contrast, the donor/acceptor rhodacarbenoid formed under otherwise identical conditions from 4-aryl-1tosyl-1,2,3-triazoles reacted with allenols through $C$ attack. Please, see reference 6.

[11] See Computational Details in the Supporting Information.

[12] Similar early dissociated of the dirhodium catalyst has been reported, see: a) Y. Liang, H. Zhou, Z.-X. Yu, J. Am. Chem. Soc. 2009, 131, 17783; b) S. C. Schmid, I. A. Guzei, I. Fernández, J. M. Schomaker, ACS Catal. 2018, 8,7907 .

[13] Experimental procedures as well as full spectroscopic and analytical data for compounds not included in this Experimental Section are described in the Supporting Information. It contains computational details, experimental procedures, characterization data, and copies of NMR spectra for all new compounds. 


\section{UPDATES}

Chemoselectivity Switching in the Rhodium-

Catalyzed Reactions of 4-Substituted-1-sulfonyl-

1,2,3-triazoles with Allenols: Noticeable

Differences between 4-Acyl- and 4-Aryl-Triazoles

Adv. Synth. Catal. 2018, 360, Page - Page

Benito Alcaide, * Pedro Almendros, * Israel Fernández, Teresa Martínez del Campo, Guillermo Palop, Mireia Toledano-Pinedo, and Patricia

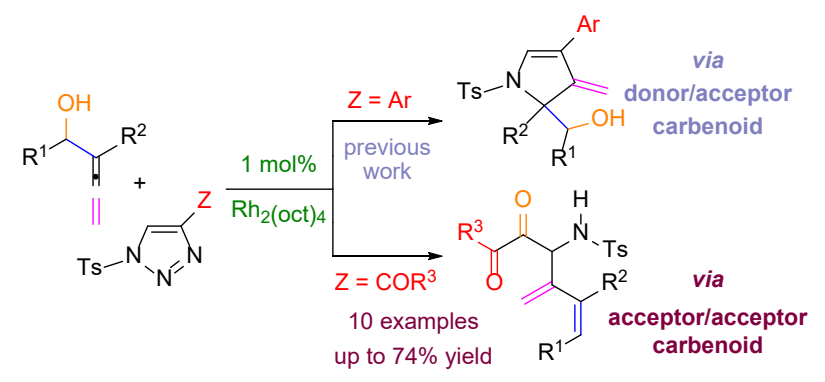
Delgado-Martínez

Autores: Alcaide, B.; Almendros, P.; Fernández, I.; Martínez del Campo, T.; Palop, G.; Toledano-Pinedo, M.; Delgado-Martínez, P.

Título: Chemoselectivity Switching in the Rhodium-Catalyzed Reactions of 4-Substituted-1-sulfonyl-1,2,3triazoles with Allenols: Noticeable Differences between 4-Acyl- and 4-Aryl-Triazoles

Revista: Adv. Synth. Catal. 2019, 361, 1160-1165; DOI: 10.1002/adsc.201801424 